\title{
Malaria epidemiology and anti-malarial drug efficacy in Guinea: a review of clinical and molecular studies
}

\author{
Mahamoud Sama Cherif ${ }^{1,2^{*}}$ (D), Prabin Dahal ${ }^{3}$ D, Abdoul Habib Beavogui ${ }^{1,2}$, Alexandre Delamou $^{1,2}$, \\ Eugene Kaman Lama ${ }^{4}$, Alioune Camara ${ }^{4}$ and Mamadou Pathe Diallo ${ }^{1}$
}

\begin{abstract}
Malaria is one of the leading causes of mortality and morbidity in Guinea. The entire country is considered at risk of the disease. Transmission occurs all year round with peaks occurring from July through October with Plasmodium falciparum as the primary parasite species. Chloroquine (CQ) was the first-line drug against uncomplicated $P$. falciparum in Guinea until 2005, prior to the adoption of artemisinin-based combination therapy (ACT). In this review, data on therapeutic efficacy of CQ and artemisinin-based combinations reported in published literature is summarized. Against CQ, a failure rate of 27\% (12/44) was reported in a study in 1992; a median failure rate of 15.6\% [range: 7.7-28.3; 8 studies] was observed during 1996-2001, and 81\% (17/21) of the patients failed to clear parasitaemia in a study conducted in 2007. For artemisinin-based combinations, three published studies were identified (1495 patients; 2004-2016); all three studies demonstrated day 28 polymerase chain reaction corrected efficacy $>95 \%$. One study characterized kelch-13 mutations (389 tested; samples collected in 2016) with no evidence of mutations currently known to be associated with artemisinin resistance. The impact of the ongoing COVID-19 pandemic and widespread usage of counterfeit medicines are immediate challenges to malaria control activities in Guinea.
\end{abstract}

Keywords: Malaria, Guinea, Resistance, Artemisinin-based combination therapy, Efficacy, Plasmodium falciparum

\section{Background}

Malaria is one of the leading causes of mortality and morbidity in Guinea with the entire population at risk of the disease [1]. In 2016, malaria was responsible for $31 \%$ of all out-patient consultations [2]. Malaria control has remained one of the top-most public-health priorities. There has also been a recent expansion in international support towards malaria control; Guinea received a funding of 30 million USD in 2017 and a further 45 million USD in 2019 [3]. The increased funding has led to a distribution of 2.7 million courses of artemisinin-based

\footnotetext{
*Correspondence: msamafr@gmail.com

${ }^{1}$ Faculty of Sciences and Health Technics, Gamal Abdel Nasser University of Conakry, Conakry, Guinea
}

Full list of author information is available at the end of the article combination therapy (ACT) in 2017 and a further 1.8 million courses in 2018 [3]. Just under 3 million rapid diagnostic tests (RDTs) were distributed annually (20172019), and long-lasting insecticidal nets (LLINs) and/or indoor residual spraying (IRS) coverage was between 50 and $80 \%$ in 2019 [3]. There is currently a surplus of insecticide-treated nets (ITNs), anti-malarial drugs, and RDTs in combatting malaria for the next 2 years [4]. Despite these important achievements, malaria remains a major public health problem. The disease burden is still high with an estimated 3.8 million cases and 8180 malariaattributed deaths in 2018 (Fig. 1A. B) [3].

This review discusses the overall epidemiological features of malaria in Guinea, presents the evolution of national malaria control programme (NMCP), highlights major milestones achieved, summarizes clinical and

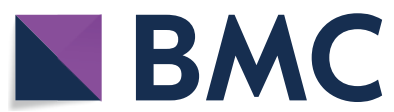

(c) The Author(s) 2021. This article is licensed under a Creative Commons Attribution 4.0 International License, which permits use, sharing, adaptation, distribution and reproduction in any medium or format, as long as you give appropriate credit to the original author(s) and the source, provide a link to the Creative Commons licence, and indicate if changes were made. The images or other third party material in this article are included in the article's Creative Commons licence, unless indicated otherwise in a credit line to the material. If material is not included in the article's Creative Commons licence and your intended use is not permitted by statutory regulation or exceeds the permitted use, you will need to obtain permission directly from the copyright holder. To view a copy of this licence, visit http://creativeco mmons.org/licenses/by/4.0/. The Creative Commons Public Domain Dedication waiver (http://creativecommons.org/publicdomain/ zero/1.0/) applies to the data made available in this article, unless otherwise stated in a credit line to the data. 

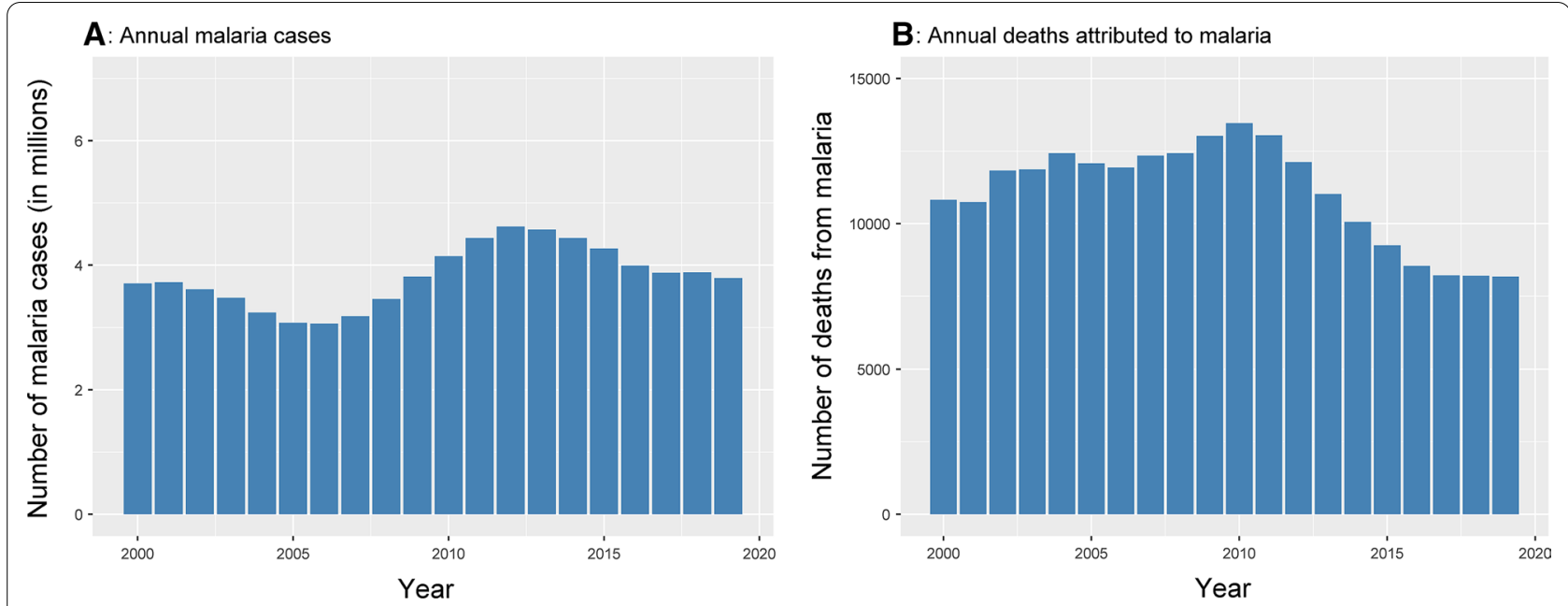

Fig. 1 Total malaria cases and deaths in Guinea. Data are from WHO World Malaria Report 2020 [3]

molecular data from published literature, and discusses some immediate challenges and future areas of focus.

\section{Epidemiological features of malaria in Guinea Parasite species}

Plasmodium falciparum is the primary cause of malaria in Guinea. Non-falciparum malaria is rare with sporadic descriptions in published literature $[5,6]$. Cases of Plasmodium vivax, Plasmodium ovale, and mixed P. falciparum and $P$. vivax infections have been reported among travellers [5, 7-9].

\section{Transmission}

The country is divided into four main ecological regions: Lower Guinea lies in the west along the coast, the Middle Guinea is a mountainous region with cooler temperature, the Upper Guinea lies to the northeast and the Forest Guinea is located in the southeast [10]. There is a regional variation in malaria endemicity with hyperendemic transmission in the southern forested region [5], and holo-endemic transmission in the lower and eastern region [10]. Malaria transmission occurs all year round with peaks occurring from July through October.

\section{Vector distribution}

The main vectors responsible for malaria transmission are Anopheles funestus, Anopheles gambiae, Anopheles arabiensis with transmission occurring from dusk to dawn [11]. Anopheles gambiae was found to be the main vector in Fouta Djallon (Middle Guinea) in the 1980s [12]. In the Forest Guinea region, An. gambiae sensu lato (s.l.) is the main vector followed by An. funestus [10]. In Conakry, An. gambiae sensu stricto (s.s.) remains the most abundant vector [13].

\section{Risk factors}

Children under the age of five bear the largest burden of the disease in the country. A 2014 nationwide crosssectional survey estimated an overall prevalence of $44 \%$ (range: 38-61\%) among children less than 9 years [5]. Risk factors include: those living in the Forest Guinea region or in rural areas, older children looked after by farmers or housewives, and those with splenomegaly [5]. Among pregnant women, risk factors include: those not using LLINs, those with sub-optimal antenatal care visits and taking incomplete sulfadoxine-pyrimethamine (SP) doses [14]. There is also a high prevalence of malaria among infants $(21.7 \%$ among infants less than 6 months old [15]).

\section{Burden of the disease}

An estimated 3.7 million cases occurred in 2000 and the annual case burden has remained approximately constant in the ensuing decades (Fig. 1) [3].

\section{Chronology of malaria control efforts in Guinea}

The Guinean government drafted the first ever policy to combat malaria in 1970 and chloroquine (CQ) was formally adopted as the first line therapy (Table 1 ). In the 1970 s, vector control measures were introduced with an overall aim of eradicating malaria from the country. In the 1980s, Guinea adopted an integrated project to fight against communicable childhood diseases (including malaria). The national guidelines for treating malaria was also updated during this decade and CQ was adopted as a prophylactic treatment in pregnant women. In the 1990s, emerging signs of resistance against CQ were observed $[6,16,17]$. Following the Abuja Summit of 2000, Guinea developed its first strategic plan (2001-2005) to achieve 
Table 1 Major milestones in malaria control activities in Guinea

\begin{tabular}{|c|c|}
\hline Years & Milestones \\
\hline 1958 & Guinea gained independence \\
\hline 1970 & $\begin{array}{l}\text { Development of a policy document to fight against malaria } \\
\text { Chloroquine (CQ) introduced as a front-line drug } \\
\text { Implementation of spraying of insecticides as a vector control measure }\end{array}$ \\
\hline 1975 & Entomological and parasitological studies conducted in Bentourayah and Béréiré in Coyah and Forécariah with WHO's support \\
\hline 1987 & $\begin{array}{l}\text { Integrated project to fight against communicable childhood diseases (CCCD) including malaria introduced in the health districts of Cona- } \\
\text { kry, Kindia and Télimélé } \\
\text { CQ adopted as a prophylaxis in pregnant women }\end{array}$ \\
\hline 2001 & $\begin{array}{l}\text { Development of national policy against malaria on the recommendations of the Abuja Summit in } 2000 \\
\text { The first strategic plan (2001-2005) drafted with the aim to reduce morbidity and mortality in children under } 5 \text { by } 50 \%\end{array}$ \\
\hline 2003 & Creation of the national programme to fight against malaria \\
\hline 2004-2005 & A randomised trial assessing the efficacy of ACTs conducted in Dabola [18] \\
\hline 2005 & $\begin{array}{l}\text { Adoption of ACT as the first-line treatment of uncomplicated falciparum malaria } \\
\text { SP adopted as intermittent preventive treatment (IPT) in pregnant women } \\
\text { The second strategic plan (2006-2010) drafted with aim of scaling up ACTs, RDTs, and LLINs }\end{array}$ \\
\hline 2008 & Revision and adoption of the national policy document for the fight against malaria \\
\hline 2009 & Implementation of the first mass distribution campaign for LLINS \\
\hline 2011 & Guinea is part of the US President's malaria initiative (US PMI) \\
\hline $2012-2016$ & $\begin{array}{l}\text { The third strategic plan (2013-2017) drafted with the aim of scaling up to the community level diagnosis using the RDTs, the management } \\
\text { of uncomplicated malaria by ACT, and severe cases using artemisinin derivatives } \\
\text { Two national campaigns for the mass distribution of LLINs carried out } \\
\text { The strengthening of IPT and the implementation of two chemoprevention campaigns }\end{array}$ \\
\hline
\end{tabular}

Source: National malaria control programme of Guinea [61]

$A C T$ artemisinin-based combination therapy, RDTs Rapid diagnostic tests, LLINs long-lasting insecticidal nets, SP sulfadoxine-pyrimethamine, CQ chloroquine

the Abuja targets of reducing morbidity and mortality in children less than 5 years by $50 \%$-an objective that eventually remained unfulfilled. After organizational restructuring, the national malarial control programme (NMCP) was formally created in 2003 with the mission to implement policies to combat malaria burden.

During the 2000s, CQ resistance was rampant and widespread (as described by Bonnet et al. [18]). In the wake of the relentless CQ resistance, two studies in Central Guinea tested the field efficacy of artemisinin-based combinations under the auspices of Médecins Sans Frontières [18]. The studies evaluated efficacy of artesunate + amodiaquine (AS $+\mathrm{AQ})$ and artesunate $+\mathrm{SP}$ $(\mathrm{AS}+\mathrm{SP})$; both these regimens were found to be highly efficacious. These results led to Guinea formally adopting artemisinin-based combination therapy (ACT) in 2005 as the first-line treatment for uncomplicated falciparum malaria. In addition, SP replaced CQ for intermittent preventive treatment (IPT) in pregnant women.

A second strategic plan (2006-2010) was adopted following the 2006 Abuja declaration with a target to achieve universal access to basic care. This led to scalingup of nationwide distribution of ACT, rapid diagnostic tests (RDTs) and LLINs through the support of Global Fund. During 2010-2019 period, "test before treat" programme was adopted and scaled up, two national campaigns of mass distribution of LLINs were carried out (in 2013 and 2016), and two seasonal malaria chemoprevention programme were also implemented. Over 27.6 million pyrethroid-treated LLINs were distributed (2013-2017) [19]. In 2011, Guinea was added to the United States' President's Malaria Initiative (US PMI) list of high malaria burden countries-the US PMI has provided critical technical and financial support in combatting the high burden of malaria.

\section{Therapeutic efficacy studies against uncomplicated $P$. falciparum malaria: the past and the present}

Relevant studies describing anti-malarial drug efficacy in Guinea were identified by searching the publications indexed in the WorldWide Antimalarial Resistance Network (WWARN) library of clinical studies [20]. The WWARN library is a periodically updated living systematic review that indexes all published anti-malarial trials from 1946 onwards. Additional studies were identified by searching the references of the included studies and by conducting further search on PubMed to identify more recent studies using a broad search terms of (malaria) AND (Guinea). Information on early and late parasitological responses were extracted from eligible studies (Table 2). Clinical efficacy of anti-malarial drugs used in the past (Chloroquine era: 1970-2005) and the present (ACT era: 2005-date) is summarized next. 


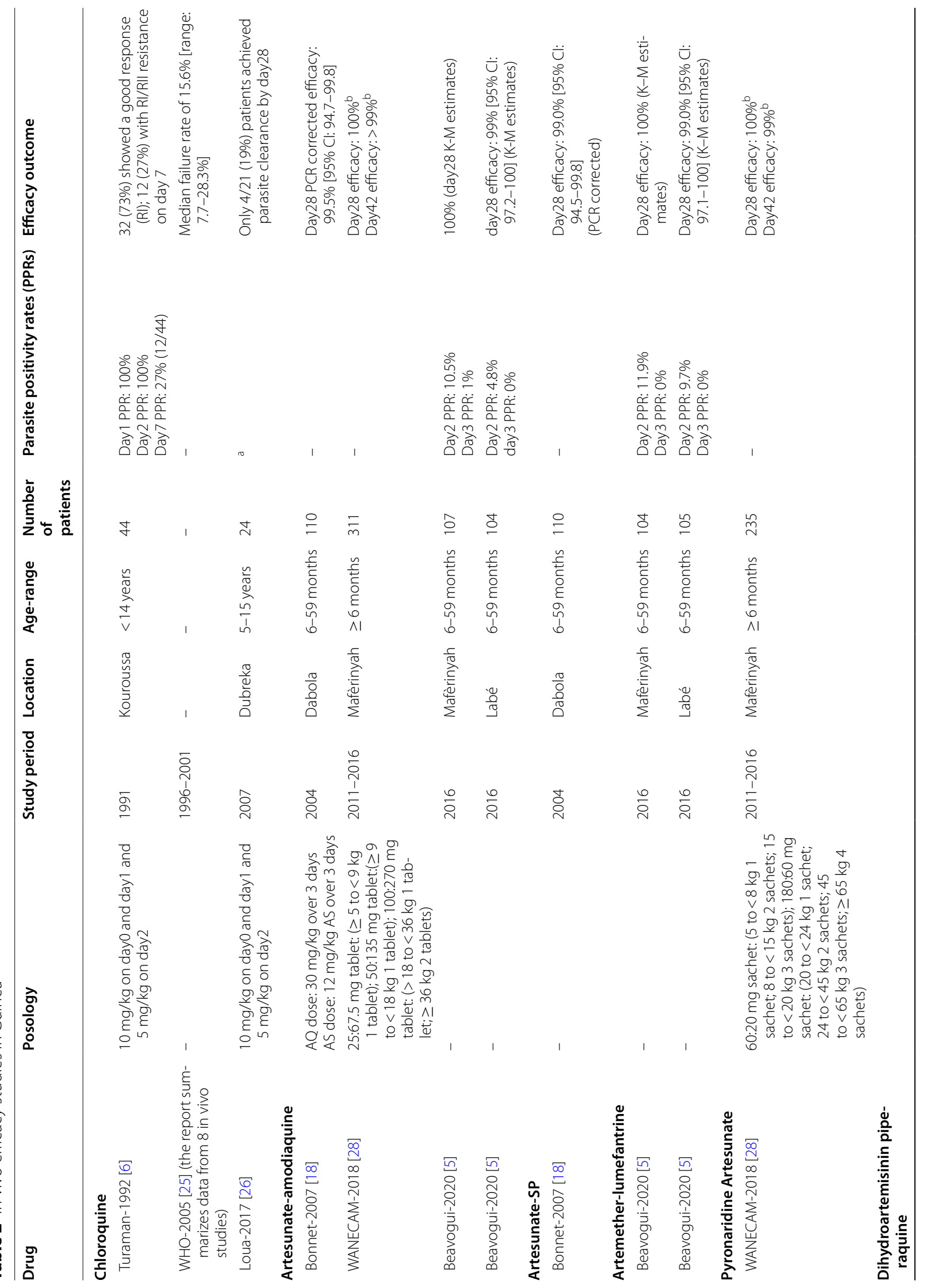




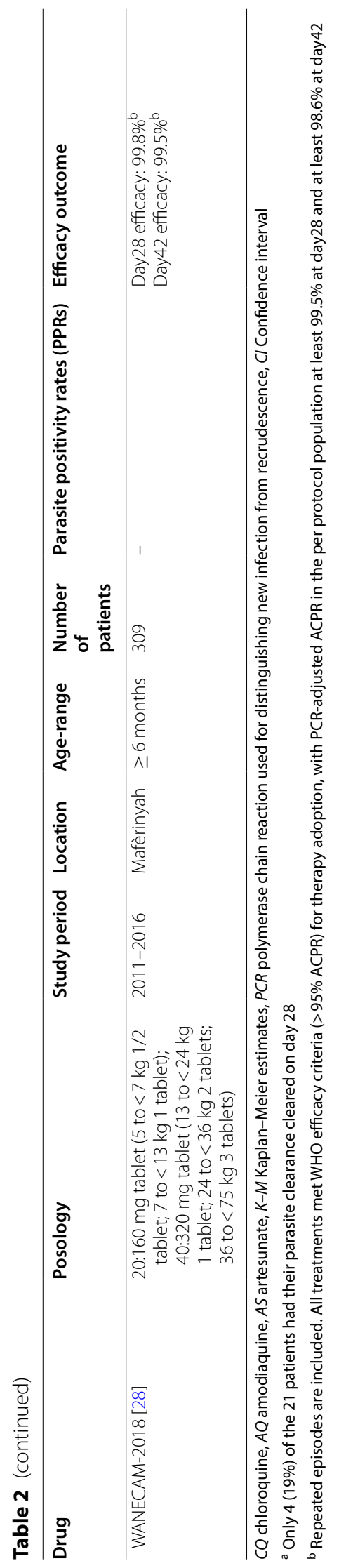




\section{Chloroquine}

CQ was the mainstay treatment against uncomplicated malaria in the 1960s. Despite reports of decreased CQ susceptibility in the neighbouring countries during the 1960 s, $P$. falciparum infections were found to respond well to CQ therapy in Guinea [21,22] and the regimen was adopted as the frontline drug in the first malaria policy document in 1970 (Table 1). By the late 1980s, there was a sharp decline in parasite susceptibility to CQ (in parallel, CQ resistance was confirmed in some West African countries) [23]. Several clinical studies conducted in Kindia and in Conakry in the 1990s found no evidence of CQ resistance when patients were treated with $25 \mathrm{mg} / \mathrm{kg}$ CQ dose over 3 days [24]. Although in vitro studies had identified resistant isolates of $P$. falciparum during late 1980s and early 1990s, there was no in vivo evidence of CQ resistance $[6,16,17]$. Evidence of partial CQ resistance emerged in 1991 in a study conducted among 44 children at Kouroussa district hospital [6]. During 19962001, 8 in vivo studies were conducted to assess the efficacy of CQ and a median failure rate of $15.6 \%$ [range: 7.7-28.3] was observed [25]. By the turn of the millennium, failure rates against CQ were greater than $20 \%$ (described by Bonnet et al. in 2006 [18]). Lack of feasible alternatives meant that $\mathrm{CQ}$ continued to be used as the first-line drug against $P$. falciparum into the early 2000 s. A study in 2007 reported that only 4/21 (19\%) patients achieved parasite clearance by day 28 after $25 \mathrm{mg} / \mathrm{kg} C Q$ dose [26]. The drug is no longer recommended after the adoption of ACT as first-line therapy.

\section{Artemisinin-based combination therapy}

The artesunate + SP $(\mathrm{AS}+\mathrm{SP})$ regimen was one of the early artemisinin-based combinations used in Guinea. It was used in the Lainé refugee camp in the forest region for over two years in the early 2000s [18]. The regimen was also tested in a clinical study of 110 patients in Dabola (2004) with a day 28 polymerase chain reaction (PCR) corrected efficacy of $99.0 \%$ [95\% confidence interval (CI): 94.5-99.8] (Table 2).

ACT was formally adopted as the first-line treatment for uncomplicated malaria by the NMCP in 2005, with artesunate + amodiaquine $(\mathrm{AS}+\mathrm{AQ})$ as the preferred regimen [27]. The efficacy of AS $+\mathrm{AQ}$ was initially tested in Dabola (2004) and has been further evaluated in Mafèrinyah (2011-2016) and Labé (2016) in a total of 632 patients (Table 2) [18, 28, 29]. Rapid parasitological responses has been observed following this regimen with day 3 parasite positivity rate (PPR) of $1 \%$ in Mafèrinyah and 0\% in Labé in 2016 (PPRs were not reported in the Dabola study).

Artemether-lumefantrine (AL) has now largely replaced AS $+\mathrm{AQ}$ as the preferred regimen since 2016
[29]. The efficacy of the AL regimen has been evaluated in a single study in 2016 (209 patients) at two sites (Mafèrinyah and Labé) [5]. The PCR corrected efficacy on day 28 at both sites were $\geq 99 \%$ and parasite positivity rates were approximately $10 \%$ on day 2 and $0 \%$ on day 3 (Table 2).

Other artemisinin-based combinations tested include pyronaridine-artesunate (PA) $(\mathrm{n}=235$ patients $)$ and dihydroartemisinin-piperaquine (DP) ( $\mathrm{n}=309$ patients) evaluated in Mafèrinyah (2011-2016) with a day 28 PCR corrected efficacy $>95 \%$ for both regimens (Table 2 ).

\section{Other anti-malarial drugs}

Quinine is now mainly used as a rescue therapy as outlined in the NMCP protocol [30]. Low sensitivity against quinine was reported in 10 isolates tested in 1986 [16] and in a traveller in 2004 [31]. Current therapeutic efficacy or drug resistance status on quinine remains unknown.

Sulfadoxine-pyrimethamine remains the current firstline therapy for treatment of malaria in pregnancy [3] and is also adopted as intermittent preventive treatment (IPT) among pregnant women [30]. Further data on in vivo efficacy of SP is not available. However, there were no known reports of resistance against SP in Guinea until 2004 (See Table 1.3 in thesis by Amin A.A. [32]). Mutations in dihydrofolate reductase ( $d h f r)$ and dyhydropteroate synthetase (dhps) genes in isolates tested after 2004 is presented in Table 3.

\section{Therapeutic efficacy studies against severe P. falciparum malaria}

The estimated incidence of in-patient severe malaria in Guinea is 115.6 cases per 100,000 person per year [33]. No published studies describing the efficacy of antimalarial drugs against severe malaria in Guinea were identified. Parenteral quinine was the first-line regimen for the treatment of severe malaria prior to its replacement by artesunate therapy [3, 30]. A case of severe malaria was described in a traveller who was successfully treated with quinine [8].

\section{Therapeutic efficacy studies against non-falciparum malaria}

There is no specific policy for the treatment of vivax or other non-falciparum malaria [3]. Reports of non-falciparum malaria are rare and are described mostly among travellers. CQ and primaquine therapy was used for treating a case of traveller vivax malaria with good clinical outcome [9]. 
Table 3 Studies describing prevalence of molecular markers of antimalarial resistance

\begin{tabular}{|c|c|c|c|c|c|c|}
\hline Markers & Year & Study site & Marker type & Total tested & $\begin{array}{l}\text { Number of } \\
\text { mutations }\end{array}$ & $\begin{array}{l}\text { Mutation percentage } \\
{[95 \% \mathrm{Cl}]}\end{array}$ \\
\hline \multicolumn{7}{|l|}{ dhps } \\
\hline Bonnet-2007 [18] & 2004 & Lainé refugee camp & Wild-type & 114 & 8 & $7.0 \%$ [3.6-13.2\%] \\
\hline Bonnet-2007 [18] & 2004 & Lainé refugee camp & Single mutant 436 & 114 & 31 & $27.2 \%$ [19.9-36\%] \\
\hline Bonnet-2007 [18] & 2004 & Lainé refugee camp & Single mutant 437 & 114 & 38 & $33.3 \%$ [25.3-42.4\%] \\
\hline Bonnet-2007 [18] & 2004 & Lainé refugee camp & Double mutant 436-437 & 114 & 29 & $25.4 \%$ [18.3-34.1\%] \\
\hline Bonnet-2007 [18] & 2004 & Lainé refugee camp & Double mutant 437-540 & 114 & 8 & $7.0 \%[3.6-13.2 \%]$ \\
\hline Xu-2019 [36] & 2013-2016 & Travellers & $1431 \mathrm{~V}$ & 13 & 0 & $0.0 \%$ [0.0-22.8\%] \\
\hline Xu-2019 [36] & 2013-2016 & Travellers & $\mathrm{S} 436 \mathrm{~A} / \mathrm{F}$ & 13 & 1 & $7.7 \%[1.4-33.3 \%]$ \\
\hline Xu-2019 [36] & 2013-2016 & Travellers & A437G & 13 & 13 & $100 \%$ [77.2-100\%] \\
\hline Xu-2019 [36] & 2013-2016 & Travellers & $\mathrm{K} 540 \mathrm{E}$ & 13 & 2 & $15.4 \%[4.3-42.2 \%]$ \\
\hline Xu-2019 [36] & 2013-2016 & Travellers & A581G & 13 & 0 & $0.0 \%[0.0-22.8 \%]$ \\
\hline Xu-2019 [36] & 2013-2016 & Travellers & A613S & 13 & 1 & $7.7 \%[1.4-33.3 \%]$ \\
\hline \multicolumn{7}{|l|}{$d h f$} \\
\hline Bonnet-2007 [18] & 2004 & Lainé refugee camp & Wild-type & 148 & 20 & $13.5 \%[8.9-20.0 \%]$ \\
\hline Bonnet-2007 [18] & 2004 & Lainé refugee camp & Double mutant 59-108 & 148 & 2 & $1.4 \%[0.4-4.8 \%]$ \\
\hline Bonnet-2007 [18] & 2004 & Lainé refugee camp & Triple mutant 51-59-108 & 148 & 126 & $85.1 \%$ [78.5-90.0\%] \\
\hline Xu-2019 [36] & 2013-2016 & Travellers & N511 & 13 & 12 & $92.3 \%[66.7-98.6 \%]$ \\
\hline $\mathrm{Xu}-2019[36]$ & 2013-2016 & Travellers & C59R & 13 & 12 & $92.3 \%[66.7-98.6 \%]$ \\
\hline Xu-2019 [36] & 2013-2016 & Travellers & $\mathrm{S} 108 \mathrm{~N}$ & 13 & 13 & $100 \%[77.2-100 \%]$ \\
\hline Xu-2019 [36] & 2013-2016 & Travellers & $511-59 R-108 \mathrm{~N}$ & 13 & 12 & $92.3 \%$ [66.7-98.6\%] \\
\hline \multicolumn{7}{|l|}{ dhps and dhfr combined } \\
\hline Bonnet-2007 [18] & 2004 & Lainé refugee camp & $\begin{array}{l}\text { Quintuple mutant: dhfr 51-59- } \\
108 \text { and dhps 437-540 }\end{array}$ & 110 & 8 & $7.3 \%[3.7-13.7 \%]$ \\
\hline Bonnet-2007 [18] & 2004 & Lainé refugee camp & $\begin{array}{l}\text { Quintuple mutant: dhfr 51-59- } \\
108 \text { and dhps 436-437 }\end{array}$ & 110 & 27 & $24.5 \%[17.5-33.4 \%]$ \\
\hline Bonnet-2007 [18] & 2004 & Lainé refugee camp & $\begin{array}{l}\text { dhfr 51-59-108 and dhps single } \\
\text { mutant }\end{array}$ & 110 & 54 & $49.1 \%$ [39.9-58.3\%] \\
\hline Bonnet-2007 [18] & 2004 & Lainé refugee camp & Three or less mutations & 110 & 21 & $19.1 \%[12.8-27.4 \%]$ \\
\hline Xu-2019 [36] & 2013-2016 & Travellers & $511+59 R+108 N+437 G(I R N G)$ & 13 & 8 & $61.5 \%[35.5-82.3 \%]$ \\
\hline Xu-2019 [36] & 2013-2016 & Travellers & $\begin{array}{l}511+59 R+108 N+437 G+540 E \\
\quad(\text { IRNGE) }\end{array}$ & 13 & 2 & $15.4 \%[4.3-42.2 \%]$ \\
\hline Xu-2019 [36] & 2013-2016 & Travellers & $\begin{array}{l}511+59 R+108 \mathrm{~N}+437 \mathrm{G}+540 \mathrm{E} \\
\quad+581 \mathrm{G} \text { or } 613 \mathrm{~S}(\text { IRNGEG/S) }\end{array}$ & 13 & 0 & $0.0 \%[0.0-22.8 \%]$ \\
\hline \multicolumn{7}{|l|}{ pfcrt } \\
\hline Durand-2001 [38] & 1995-1999 & Travellers & pfcrt 76T & 1 & 0 & $0.0 \%[0.0-79.3 \%]$ \\
\hline Durand-2001 [38] & 1995-1999 & Travellers & pfcrt K76 & 1 & 1 & $100.0 \%$ [20.7-100\%] \\
\hline Andriantsoanirina-2010 [37] & 2003 & Travellers & pfcrt 76T & 1 & 1 & $100.0 \%$ [20.7-100\%] \\
\hline Andriantsoanirina-2010 [37] & 2003 & Travellers & pfcrt K76 & 1 & 0 & $0.0 \%[0.0-79.3 \%]$ \\
\hline Zhou-2016 [39] & 2012-2015 & Conakry & pfcrt CMNK & 33 & 21 & $63.6 \%[46.6-77.8 \%]$ \\
\hline Zhou-2016 [39] & 2012-2015 & Conakry & pfcrt CIET & 33 & 11 & $33.3 \%$ [19.8-50.4\%] \\
\hline Zhou-2016 [39] & 2012-2015 & Conakry & pfcrt K76 & 33 & 21 & $63.6 \%[46.6-77.8 \%]$ \\
\hline Zhou-2016 [39] & 2012-2015 & Conakry & pfcrt 76T & 33 & 11 & $33.3 \%[19.8-50.4 \%]$ \\
\hline Lu-2017 [40] & $2011-2014$ & - & pfcrt K76 & 7 & 3 & $42.9 \%[15.8-75.0 \%]$ \\
\hline Lu-2017 [40] & $2011-2014$ & - & pfcrt 76T & 7 & 4 & $57.1 \%[25.0-84.2 \%]$ \\
\hline Lu-2017 [40] & $2011-2014$ & - & pfcrt 76K/T & 7 & 0 & $0.0 \%[0.0-35.4 \%]$ \\
\hline Lu-2017 [40] & 2011-2014 & - & pfcrt CMNK & 7 & 3 & $42.9 \%[15.8-75.0 \%]$ \\
\hline Lu-2017 [40] & $2011-2014$ & - & pfcrt CIET & 7 & 4 & $57.1 \%$ [25.0-84.2\%] \\
\hline Tao-2018 [41] & 2012-2016 & Conakry & pfcrt 76T & 10 & 3 & $30.0 \%$ [10.8-60.3\%] \\
\hline Tao-2018 [41] & $2012-2016$ & Conakry & pfcrt K76 & 10 & 7 & $70.0 \%$ [39.7-89.2\%] \\
\hline \multicolumn{7}{|l|}{ pfmdr1 } \\
\hline Witkowski-2010 [43] & 2005-2009 & Travellers & Copy number $>1$ & 9 & 0 & $0.0 \%[0.0-29.9 \%]$ \\
\hline Durand-2001 [38] & 1995-1999 & Travellers & pfmdr1 86Y & 1 & 1 & $100.0 \%$ [20.7-100\%] \\
\hline Durand-2001 [38] & 1995-1999 & Travellers & pfmdr1 N86 & 1 & 0 & $0.0 \%$ [0.0-79.3\%] \\
\hline
\end{tabular}


Table 3 (continued)

\begin{tabular}{|c|c|c|c|c|c|c|}
\hline Markers & Year & Study site & Marker type & Total tested & $\begin{array}{l}\text { Number of } \\
\text { mutations }\end{array}$ & $\begin{array}{l}\text { Mutation percentage } \\
{[95 \% \mathrm{Cl}]}\end{array}$ \\
\hline Tao-2018 [41] & $2012-2016$ & Conakry & pfmdr1 86Y & 10 & 2 & $20.0 \%[5.7-51.0 \%]$ \\
\hline Yang-2019 [42] & 2012-2016 & Conakry & pfmdr1 NFSND & 33 & 14 & $42.4 \%$ [27.2-59.2\%] \\
\hline Yang-2019 [42] & 2012-2016 & Conakry & pfmdr1 YYSNY & 33 & 0 & $0.0 \%[0.0-10.4 \%]$ \\
\hline Yang-2019 [42] & 2012-2016 & Conakry & pfmdr1 N86 & 33 & 14 & $42.4 \%[27.2-59.2 \%]$ \\
\hline Yang-2019 [42] & 2012-2016 & Conakry & pfmdr1 $86 Y$ & 33 & 19 & $57.6 \%[40.8-72.8 \%]$ \\
\hline Beavogui-2020 [5] & 2016 & Mafèrinyah and Labé & pfmdr1 86N & 379 & 296 & $78.1 \%[73.7-82 \%]$ \\
\hline Beavogui-2020 [5] & 2016 & Mafèrinyah and Labé & pfmdr1 86Y & 379 & 59 & $15.6 \%$ [12.3-19.6\%] \\
\hline Beavogui-2020 [5] & 2016 & Mafèrinyah and Labé & pfmdr1 86N/Y & 379 & 24 & $6.3 \%[4.3-9.2 \%]$ \\
\hline Beavogui-2020 [5] & 2016 & Mafèrinyah and Labé & pfmdr1 184Y & 379 & 117 & $30.9 \%$ [26.4-35.7\%] \\
\hline Beavogui-2020 [5] & 2016 & Mafèrinyah and Labé & pfmdr1 $184 \mathrm{~F}$ & 379 & 206 & $54.4 \%$ [49.3-59.3\%] \\
\hline Beavogui-2020 [5] & 2016 & Mafèrinyah and Labé & pfmdr1 184 Y/F & 379 & 56 & $14.8 \%$ [11.6-18.7\%] \\
\hline Beavogui-2020 [5] & 2016 & Mafèrinyah and Labé & pfmdr1 1246 D & 370 & 363 & $98.1 \%$ [96.1-99.1\%] \\
\hline Beavogui-2020 [5] & 2016 & Mafèrinyah and Labé & pfmdr1 1246Y & 370 & 6 & $1.6 \%[0.7-3.5 \%]$ \\
\hline Beavogui-2020 [5] & 2016 & Mafèrinyah and Labé & pfmdr1 $1246 \mathrm{D} / \mathrm{Y}$ & 370 & 1 & $0.3 \%[0.0-1.5 \%]$ \\
\hline \multicolumn{7}{|l|}{ pfk13 } \\
\hline Beavogui-2020 [5] & 2016 & Mafèrinyah and Labé & Wild-type & 389 & 380 & $97.7 \%$ [95.7-98.8\%] \\
\hline Beavogui-2020 [5] & 2016 & Mafèrinyah and Labé & Mutant & 389 & 9 & $2.3 \%$ [1.2-4.3\%] \\
\hline
\end{tabular}

Source: The table is derived from the open-access library of molecular markers of antimalarial resistance indexed in the WorldWide Antimalarial Resistance Network systematic review libraries [34, 35]

$\mathrm{Cl}$ confidence interval; $95 \% \mathrm{Cl}$ was derived using Wilson's method

\section{Studies describing molecular markers of drug resistance}

The WorldWide Antimalarial Resistance Network (WWARN) database of molecular markers of resistance against partner drugs for ACT and for SP were searched for identifying publications from Guinea [34, 35]. A recently published study was identified from a further literature search carried out by using the search terms of (malaria) AND (Guinea) on PubMed [5]. Reported mutations are summarized in Table 3 and some key results are presented below.

\section{Dihydrofolate reductase (dhfr) and dihydropteroate synthetase (dhps)}

In 2004, single or double mutations in dhps 436-437 or dhps 437-540 were found in 93\% (106/114) and $d h f r$ $59-108$ or dhfr 51-59-108 mutations in $86.5 \%(128 / 148)$ of the isolates tested in the Laine refugee camp [18]. Molecular data from 13 travellers (2013-2016) indicated 92.3\% (12/13) harboured triple dhfr (51-59-108) mutations [36]. All the isolates also harboured single mutation in dhps 437 gene with dhps 436 present in $7.7 \%(1 / 13)$ and dhps 540 in $15.4 \%(2 / 13)$.

\section{Plasmodium falciparum chloroquine resistance transporter (pfcrt)}

pfcrt K76 was identified in travellers in the late 1990s and early 2000s [37, 38]. During 2012-2015, a study reported the presence of $p f c r t 76 \mathrm{~T}$ allele in $33.3 \%(11 / 33)$ travellers [39]. Data from travellers continue to indicate the presence of mutations in the pfcrt genes [39-42].

\section{Plasmodium falciparum multi-drug resistance 1 (pfmdr1)}

pfmdr1 N86 mutations were reported in 78.1\% (298/379) of the isolates tested in a clinical study carried out in Mafèrinyah and Labé in 2016 [5] (Table 3). Among travellers, a study reporting data from 2005-2009 found no copy number elevations in $p f m d r 1$ gene [43]. Another study reported $p f m d r 1$ N86 mutations in $42.4 \%(14 / 33)$ of the travellers (2012-2016) [42].

\section{Plasmodium falciparum kelch-13 (pfk13)}

In 2016, isolates from patients in Mafèrinyah and Labé found that $2.3 \%(9 / 389)$ harboured kelch-13 mutations [5]. The reported mutations however are currently not known to be associated with resistance against artemisinin.

\section{Discussion}

Despite ACT being the first-line therapy for more than 15 years, only three published in vivo trials were identified (1495 patients, conducted from 2004 to 2016). Data from these three trials demonstrate rapid clearance of parasites and an efficacy greater than 95\%-as expected for ACT [44-48]. In particular, the three 
studies described data collected up to 2016 and there is a paucity of in vivo parasite susceptibility from 2017 onwards. The NMCP has begun therapeutic efficacy studies in four sentinel sites to monitor drug efficacy and track early signs of drug resistance [29].

In contrast to the paucity of data on drug efficacy, studies describing molecular markers were relatively more common (Table 3). Mutations were reported in pfmdr1 N86 in 78.1\% (298/379) from Mafèrinyah and Labé in 2016 [5]. Mutation in pfmdr1 allele is associated with increased failure for ACT with lumefantrine as partner drug [49]. Monitoring mutations in pfmdr1 gene including assessment of copy number elevation is, therefore, critical for the Guinean malaria control since AL has now largely replaced AS + AQ as a front line drug [29]. One study described mutations in the kelch-13 gene [5] and reassuringly, all the mutations identified are currently known not be associated with artemisinin resistance.

In the past 20 years, Guinea has made important progress towards combatting malaria, including implementation of several mass drug administration campaigns, distribution of LLINs, improved access of RDTs and ACT. All these achievements are yet to materialize in a visible reduction in case burden; approximately 3 million cases still occur annually. A major challenge is that despite improved treatment access and coverage, the adoption of ACT could be actually sub-optimal. Among children less than 5 years of age with fever and malaria, the estimated uptake of ACT was only 1.2\% [95\% CI: $0.6-2.1 \%$ ] in 2015 [50]. Awareness campaigns and public/community engagement activities might, therefore, be needed [51]. An integrated approach that focuses on overall aspect of malaria epidemiology, including vector control measures such as promotion of environmental sanitation to reduce the breeding sites for mosquitoes in addition to the periodic assessment of drug efficacy, and continuous surveillance of molecular markers across wide geographic span is likely to be key to reducing the overall case burden.

There are also several immediate challenges. First, the ongoing COVID-19 pandemic remains a major immediate threat. Unlike the 2014-2015 Ebola Virus Disease (EVD) outbreak that devastated control activities only in some regions, the ongoing COVID-19 pandemic has disrupted control activities throughout the country. The number of suspected malaria cases has increased by $2.6 \%$ (from 3,334,355 in 2019 to 3,422,309 in 2020) and the number of rapid diagnostic test (RDTs) confirmed positive cases has increased by 10.9\% (from 1,801,694 in 2019 to $1,998,329$ in 2020) (Eugene Kaman Lama, NMCP, pers. commun.). The full impact of the pandemic on malaria control activities cannot be clearly assessed at this moment, as the future course of the pandemic remains unknown. For example, during the 2014-2015 EVD outbreak, there was an estimated $45 \%$ increase in untreated malaria cases and 5,600 [95\% CI: 3000-11,100] additional malaria attributed deaths [52]. Although the current data has shown only moderate increase in malaria cases (in 2020 compared to 2019), the impact is likely to be substantial if the ongoing COVID-19 pandemic leads to persistent interruption of the control activities for a longer period. Second, counterfeit/sub-standard/falsified medicines is a major public health problem and has been under-recognized. Reports of fake chloroquine and halofantrine in circulation were documented in 2009 [53]. Despite several crackdowns from the government, counterfeit medicines can easily be purchased in the Niger and Madina markets in Conakry [54, 55]. The government seized more than 300 containers of fake medicines in Conakry port in 2019-this likely indicates the scale of widespread availability of fake drugs [56]. It is reported that more than 100,000 Guineans die annually due to fake or falsified drugs [57]. Guinea is a recent signatory on a treaty for the establishment of African Medicines Agency (AMA). The AMA has an overarching objective to improve access to quality, safe and efficacious medical products throughout the continent [58]. Such collaborative initiative is important for the control of sub-standard medicines in the entire region.

Finally, the recent report of the identification of a de novo emergence of Kelch-13 mediated artemisininresistance in Rwanda is a major concern [59, 60], but a recent study conducted in Mafèrinyah and Labé has not identified any mutations that are currently known to be associated with artemisinin resistance [5]. Continuous monitoring of the therapeutic efficacy of the existing front line drugs and conducting molecular surveillance studies to generate complementary information on drug resistance remains crucial.

\section{Conclusion}

There is limited data on in vivo efficacy of ACT regimens in Guinea despite their adoption as first-line treatment for 15 years. Annual case burden still remains very high with the entire population at risk of malaria. The threat of COVID-19 pandemic and the widespread availability of counterfeit medicines remains major immediate challenges.

\footnotetext{
Abbreviations

ACT: Artemisinin-based combination therapy; AL: Artemether-lumefantrine; $\mathrm{AS}+\mathrm{AQ}$ : Artesunate-amodiaquine; $\mathrm{AS}+\mathrm{MQ}$ : Artesunate-mefloquine; $\mathrm{Cl}$ : Confidence interval; CQ: Chloroquine; DP: Dihydroartemisinin-piperaquine; IRS: Indoor residual spray; ITNs: Insecticide-treated nets; LLINs: Long-lasting insecticidal nets; NMCP: National malaria control programme; PCR: Polymerase chain reaction; RDTs: Rapid diagnostic tests; SP: Sulfadoxine-pyrimethamine;
} 
TES: Therapeutic efficacy study; WWARN: WorldWide Antimalarial Resistance Network.

\section{Acknowledgements}

We would like to thank WorldWide Antimalarial Resistance Network (WWARN) for the review databases.

\section{Authors' contributions}

Conceptualization: MSC, PD, MPD. Data Curation: MSC, PD. Formal analysis: MSC. Supervision: MPD. Visualization: PD. Writing —original draft preparation: MSC, PD, MPD. Writing — review and editing: MSC, PD, AHB, AD, EKL, AC, MPD. All authors read and approved the final manuscript.

\section{Funding}

The review was not funded.

\section{Availability of data and materials}

All the data used are available within tables and figures presented in the manuscript.

\section{Declarations}

Ethics approval and consent to participate

Not applicable.

\section{Consent for publication}

Not applicable.

\section{Competing interests}

All authors declare that they have no competing interests.

\section{Author details}

${ }^{1}$ Faculty of Sciences and Health Technics, Gamal Abdel Nasser University of Conakry, Conakry, Guinea. ${ }^{2}$ Centre National de Formation et Recherche en Sante Rurale de Maferinyah, Maferinyah, Guinea. ${ }^{3}$ Centre for Tropical Medicine and Global Health, Nuffield Department of Medicine, University of Oxford, Oxford, UK. ${ }^{4}$ National Malaria Control Programme (NMCP), Conakry, Guinea.

\section{Received: 8 February 2021 Accepted: 8 June 2021}

Published online: 16 June 2021

\section{References}

1. U.S. President's Malaria Initiative. Guinea Malaria Operational Plan FY 2020 [Internet]. pmi.gov. 2020 [cited 2020 Dec 4]. Available from: https://www. pmi.gov/where-we-work/guinea.

2. Sun Y, Guilavogui T, Camara A, Dioubaté M, Toure BD, Bahati C, et al. Evaluating the quality of routinely reported data on malaria commodity stocks in Guinea, 2014-2016. Malar J. 2018;17:461.

3. WHO. World Malaria Report 2020. Geneva: World Health Organization; 2020.

4. ww.pmi.gov. US President's Malaria Initiative Guinea: Malaria Operational Plan FY 2021 [Internet]. 2020. [cited 2020 Dec 4]. Available from: https:// www.pmi.gov/where-we-work/guinea.

5. Beavogui AH, Delamou A, Camara BS, Camara D, Kourouma K, Camara $R$, et al. Prevalence of malaria and factors associated with infection in children aged 6 months to 9 years in Guinea: results from a national cross-sectional study. Parasite Epidemiol Control. 2020;11:e00162.

6. Turaman C, Basco LK, Le Bras J. Evaluating the efficacy of chloroquine in febrile Guinean children infected with Plasmodium falciparum by a simplified in vivo test. Bull World Health Organ. 1992;70:477-80.

7. Alfandari S, Santré C, Chidiac C, Senneville E, Leroy O, Beuscart C, et al. Imported malaria: presentation and outcome of 111 cases. Clin Microbiol Infect. 1996;2:86-90.

8. Carlsson T, Bergqvist L, Hellgren U. Homeopathic resistant malaria. J Travel Med. 1996;3:62

9. Wickramage K, Galappaththy GNL. Malaria burden in irregular migrants returning to Sri Lanka from human smuggling operations in West Africa and implications for a country reaching malaria elimination. Trans R Soc Trop Med Hyg. 2013;107:337-40.

10. Carnevale P, Toto JC, Guibert P, Keita M, Manguin S. Entomological survey and report of a knockdown resistance mutation in the malaria vector Anopheles gambiae from the Republic of Guinea. Trans R Soc Trop Med Hyg. 2010;104:484-9.

11. International Association for Medical Assistance to Travelers. Guinea General Health Risks: Malaria [Internet]. 2020 [cited 2020 Dec 13]. Available from: https://www.iamat.org/country/guinea/risk/malaria\#.

12. Bosman A, Modiano D, Voglino MC, Pizzi L, Bartoloni P, Kandia Diallo I, et al. Malaria transmission in a central area of Futa Djalon (Guinea): results of a parasitological survey during the 1989 rainy season. Parassitologia. 1992;34:135-42.

13. Vezenegho SB, Brooke BD, Hunt RH, Coetzee M, Koekemoer LL. Malaria vector composition and insecticide susceptibility status in Guinea Conakry, West Africa. MedVet Entomol. 2009;23:326-34.

14. Touré AA, Doumbouya A, Diallo A, Loua G, Cissé A, Sidibé S, et al. Malariaassociated factors among pregnant women in Guinea. J Trop Med. 2019;2019:3925094

15. Ceesay SJ, Koivogui L, Nahum A, Taal MA, Okebe J, Affara M, et al. Malaria prevalence among young infants in different transmission settings, Africa. Emerg Infect Dis. 2015;21:1114-21.

16. Brasseur P, Pathe-diallo M, Druilhe P. Low sensitivity to chloroquine and quinine of Plasmodium falciparum isolates from Guinea in March 1986. Am J Trop Med Hyg. 1987;37:452-4

17. Sanguigni S, De Gregorio CG, Cultrera R, Marangi M. Chloroquineresistant falciparum malaria from Guinea. Trans R Soc Trop Med Hyg 1988;82:359.

18. Bonnet M, Roper C, Félix M, Coulibaly L, Kankolongo GM, Guthmann JP. Efficacy of antimalarial treatment in Guinea: in vivo study of two artemisinin combination therapies in Dabola and molecular markers of resistance to sulphadoxine-pyrimethamine in N'Zérékoré. Malar J. 2007;6:54.

19. Collins E, Vaselli NM, Sylla M, Beavogui AH, Orsborne J, Lawrence G, et al. The relationship between insecticide resistance, mosquito age and malaria prevalence in Anopheles gambiae s.l. from Guinea. Sci Rep. 2019;9:8846.

20. WWARN. Clinical Trials Publication Library [Internet]. 2021 [cited 2021 Feb 5]. Available from: https://www.wwarn.org/tools-resources/literaturereviews/wwarn-clinical-trials-publication-library.

21. WHO. Technical Report Series No 296: Resistance of malaria parasites to drugs. Geneva, World Health Organization, 1965.

22. WHO. Chemotherapy of malaria. Technical Report Series No. 375: Geneva, World Health Organization, 1967.

23. Payne D. Spread of chloroquine resistance in Plasmodium falciparum. Parasitol Today. 1987;3:241-6.

24. Africa Child Survival Initiative-Combatting Childhood Communicable Diseases, Centers for Disease Control, International Health Program Office, U. S. Agency for International Development. Controlling malaria in Africa : progress and priorities [Internet]. 1993. Available from: https:// stacks.cdc.gov/view/cdc/7652. Accessed 10 Jun 2021.

25. WHO. Susceptibility of Plasmodium falciparum to antimalarial drugs: report on global monitoring: 1996-2004. Geneva: World Health Organization; 2005.

26. Loua J, Traore MS, Camara A, Balde MA, Maes L, Pieters L, et al. Biological and phytochemical investigations on Caesalpinia benthamiana, a plant traditionally used as antimalarial in Guinea. Evid Based Complement Altern Med. 2017:2017:9438607.

27. Camara A, Moriarty LF, Guilavogui T, Diakité PS, Zoumanigui JS, Sidibé S, et al. Prescriber practices and patient adherence to artemisinin-based combination therapy for the treatment of uncomplicated malaria in Guinea, 2016. Malar J. 2019;18:23.

28. Sagara I, Beavogui AH, Zongo I, Soulama I, Borghini-Fuhrer I, Fofana B, et al. Pyronaridine-artesunate or dihydroartemisinin-piperaquine versus current first-line therapies for repeated treatment of uncomplicated malaria: a randomised, multicentre, open-label, longitudinal, controlled, phase 3b/4 trial. Lancet. 2018;391:1378-90.

29. Beavogui AH, Camara A, Delamou A, Diallo MS, Doumbouya A, Kourouma $K$, et al. Efficacy and safety of artesunate-amodiaquine and artemetherlumefantrine and prevalence of molecular markers associated with resistance, Guinea: an open-label two-arm randomised controlled trial. Malar J. 2020;19:223. 
30. WHO. Country antimalarial drug policies: by region [Internet]. Geneva, World Health Organization, 2020 [cited 2020 Dec 24]. Available from: https://www.who.int/malaria/am_drug_policies_by_region_afro/en/.

31. Uchiyama H, Okamoto A, Sato K, Yamada T, Murakami S, Yoneda S, et al. Quinine-resistant severe falciparum malaria effectively treated with atovaquone and proguanil hydrochloride combination therapy. Intern Med. 2004;43:624-7.

32. Amin AA. Range, quality, and costs of antimalarial drugs available in the retail sector in Kenya [Internet]. PhD Thesis, The Open University; 2005. Available from: http://oro.open.ac.uk/66018/. Accessed 10 Jun 2021.

33. Camponovo F, Bever CA, Galactionova K, Smith T, Penny MA. Incidence and admission rates for severe malaria and their impact on mortality in Africa. Malar J. 2017;16:1.

34. WorldWide Antimalarial Resistance Network. ACT Partner Drug Molecular Surveyor [Internet]. 2020 [cited 2020 Dec 24]. Available from: http://www. wwarn.org/molecular/surveyor/\#0.

35. Worldwide Antimalarial Resistance Network. SP Molecular Surveyor [Internet]. 2020 [cited 2020 Dec 24]. Available from: http://www.wwarn. org/dhfr-dhps-surveyor/\#0.

36. Xu C, Sun H, Wei Q, Li J, Xiao T, Kong X, et al. Mutation profile of pfdhfr and pfdhps in Plasmodium falciparum among returned Chinese migrant workers from Africa. Antimicrob Agents Chemother. 2019;63:e01927-e2018.

37. Andriantsoanirina V, Ménard D, Rabearimanana S, Hubert V, Bouchier C, Tichit M, et al. Association of microsatellite variations of Plasmodium falciparum $\mathrm{Na}+/ \mathrm{H}+$ exchanger (Pfnhe-1) gene with reduced in vitro susceptibility to quinine: Lack of confirmation in clinical isolates from Africa. Am J Trop Med Hyg. 2010;82:782-7.

38. Durand R, Jafari S, Vauzelle J, Delabre JF, Jesic Z, Le Bras J. Analysis of pfcrt point mutations and chloroquine susceptibility in isolates of Plasmodium falciparum. Mol Biochem Parasitol. 2001;114:95-102.

39. Zhou RM, Zhang HW, Yang CY, Liu Y, Zhao YL, Li SH, et al. Molecular mutation profile of pfcrt in Plasmodium falciparum isolates imported from Africa in Henan province. Malar J. 2016;15:265.

40. Lu F, Zhang M, Culleton RL, Xu S, Tang J, Zhou H, et al. Return of chloroquine sensitivity to Africa? Surveillance of African Plasmodium falciparum chloroquine resistance through malaria imported to China. Parasit Vectors. 2017:10:355.

41. Zhang T, Xu X, Jiang J, Yu C, Tian C, Li W. Surveillance of antimalarial resistance molecular markers in imported Plasmodium falciparum malaria cases in Anhui, China, 2012-2016. Am J Trop Med Hyg. 2018;98:1132-6.

42. Yang C, Zhou R, Liu Y, Li S, Qian D, Zhao Y, et al. Molecular investigation of the Pfmdr1 gene of Plasmodium falciparum isolates in Henan Province imported from Africa. Parasitology. 2019:146:372-9.

43. Witkowski B, Nicolau ML, Soh PN, Iriart X, Menard S, Alvarez M, et al. Plasmodium falciparum isolates with increased pfmdr 1 copy number circulate in West Africa. Antimicrob Agents Chemother. 2010;54:3049-51.

44. The WorldWide Antimalarial Resistance Network (WWARN) DP Study Group. The effect of dosing regimens on the antimalarial efficacy of dihydroartemisinin-piperaquine: a pooled analysis of individual patient data. PLoS Med. 2013;10:e1001564

45. Worldwide Antimalarial Resistance Network (WWARN) AL Dose Impact Study Group. The effect of dose on the antimalarial efficacy of artemether-lumefantrine: a systematic review and pooled analysis of individual patient data. Lancet Infect Dis. 2015;15:692-702.

46. WWARN Parasite Clearance Study Group. Baseline data of parasite clearance in patients with falciparum malaria treated with an artemisinin derivative: an individual patient data meta-analysis. Malar J. 2015;14:359.

47. The WorldWide Antimalarial Resistance Network (WWARN) AS-AQ Study Group. The effect of dosing strategies on the therapeutic efficacy of artesunate-amodiaquine for uncomplicated malaria: a meta-analysis of individual patient data. BMC Med. 2015;13:66.

48. WWARN ACT Africa Baseline Study Group. Clinical determinants of early parasitological response to ACTs in African patients with uncomplicated falciparum malaria: a literature review and meta-analysis of individual patient data. BMC Med. 2015:13:212
49. Venkatesan M, Gadalla NB, Stepniewska K, Dahal P, Nsanzabana C, Moriera C, et al. Polymorphisms in Plasmodium falciparum chloroquine resistance transporter and multidrug resistance 1 genes: parasite risk factors that affect treatment outcomes for P. falciparum malaria after artemether-lumefantrine and artesunate-amodiaquine. Am J Trop Med Hyg. 2014;91:833-43.

50. Bennett A, Bisanzio D, Yukich JO, Mappin B, Fergus CA, Lynch M, et al. Population coverage of artemisinin-based combination treatment in children younger than 5 years with fever and Plasmodium falciparum infection in Africa, 2003-2015: a modelling study using data from national surveys. Lancet Glob Health. 2017;5:e418-27.

51. Ruberto I, Camara S, Banek K, Loua MK. Knowledge, attitudes and practices of malaria control among communities from the health district of Forécariah in the Republic of Guinea, West Africa. J Vector Borne Dis. 2014:51:119-27.

52. Walker PGT, White MT, Griffin JT, Reynolds A, Ferguson NM, Ghani AC Malaria morbidity and mortality in Ebola-affected countries caused by decreased health-care capacity, and the potential effect of mitigation strategies: a modelling analysis. Lancet Infect Dis. 2015;15:825-32.

53. United Nations Office on Drugs and Crime. Transnational trafficking and the rule of law in West Africa: a threat assessment [Internet]. Vienna, Austria; 2009 [cited 2021 Jan 4]. Available from: https://www.unodc.org/ nigeria/en/transnational-trafficking-and-the-rule-of-law-in-west-afric a_-a-threat-assessment.html.

54. The New Humanitarian. Black market medicines re-emerge [Internet] 2009 [cited 2020 Dec 22]. Available from: https://www.thenewhumanitar ian.org/report/84644/guinea-black-market-medicines-re-emerge.

55. The Institute of Research Against Counterfeit Medicines. Guinea: pharmacists syndicate calls for madina crack-down, the country's largest fake medicine market [Internet]. 2020 [cited 2020 Dec 22]. Available from: http://www.iracm.com/en/2016/12/quinea-pharmacists-syndicate-callsmadina-crack-countrys-largest-fake-medicine-market/\#.

56. Guineematin.com. Imports of fake medicines in Guinea: Dr Manizé Kolié denounces the "death sellers" [Internet]. guineematin.com. 2019 [cited 2020 Dec 22]. Available from: https://guineematin.com/2019/12/19/ importations-de-faux-medicaments-en-guinee-dr-manize-kolie-denon ce-les-vendeurs-de-mort/.

57. Guineematin.com. Fake drugs in Guinea: the revelations of the Minister of Health in Parliament [Internet]. guineematin.com. 2020 [cited 2020 Dec 22]. Available from: https://guineematin.com/2020/11/11/faux-medic aments-en-guinee-les-revelations-du-ministre-de-la-sante-au-parle ment/.

58. African Medicines Agency. Treaty for the establishment of the African Medicines Agency [Internet]. Addis-Ababa, Ethiopia; 2019. Available from: https://au.int/sites/default/files/treaties/36892-treaty-0069_-_ama_ treaty_e.pdf. Accessed 22 Dec 2020.

59. Uwimana A, Legrand E, Stokes BH, Ndikumana JLM, Warsame M, Umulisa $\mathrm{N}$, et al. Emergence and clonal expansion of in vitro artemisinin-resistant Plasmodium falciparum kelch13 R561 H mutant parasites in Rwanda. Nat Med. 2020;26:1602-8.

60. Uwimana A, Umulisa N, Venkatesan M, Svigel SS, Zhou Z, Munyaneza T, et al. Association of Plasmodium falciparum kelch13 R561H genotypes with delayed parasite clearance in Rwanda: an open-label, single-arm, multicentre, therapeutic efficacy study. Lancet Infect Dis. 2021;3099:1-8.

61. National malaria control programme- Guinea (PNLP). History of the fight against malaria [Internet]. http://pnlp-guinee.org/. 2020 [cited 2020 Dec 12]. Available from: http://pnlp-guinee.org/presentation-et-organisati on/\#.

\section{Publisher's Note}

Springer Nature remains neutral with regard to jurisdictional claims in published maps and institutional affiliations. 